\title{
Identification of New Markers in Xp21 between DXS28 (C7) and DMD
}

\author{
K. C. Worley, ${ }^{*} J$. A. Towbin, ${ }^{*} †$ X. M. Zhu, ${ }^{*}$ D. F. Barker, $\neq$ A. Ballabio, ${ }^{*}$ J. Chamberlain,,

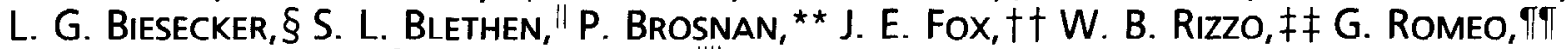 \\ N. Sakuragawa, $\S \S$ W. K. Seltzer, ${ }^{\prime l l}$ S. Yamaguchi, ${ }^{\star \star \star}$ and E. R. B. McCabe* $\dagger$
}

*Institute for Molecular Genetics and †Department of Pediatrics, Baylor College of Medicine, Houston, Texas $77030 ; \ddagger G e n e t i c$ Epidemiology, University of Utah, Salt Lake City, Utah 84108; IDepartment of Human Genetics and §Division of Pediatric Genetics, University of Michigan, Ann Arbor, Michigan 48109; "Department of Pediatrics, State University of New York, Stony Brook, New York 11794-8111; “`Driscol Foundation Children's Hospital, Corpus Christi, Texas 78466; ††Schneider Children's Hospital, New Hyde Park, New York 11042; $¥ \ddagger$ Department of Pediatrics, Medical College of Virginia, Richmond, Virginia 23298; 9 ILaboratorio di Genetica Molecolare, Instituto G Gaslini, Genova, Italy 16148; §§Division of Inherited Metabolic Diseases, National Institute of Neuroscience, National Center of Neurology and Psychiatry, Kodaira, Tokyo 187, Japan; lill University of Colorado, Denver, Colorado 80202; and ${ }^{\star \star \star}$ Gifu University School of Medicine, Gifu 500, Japan

Received December 26, 1991; revised April 8, 1992

Characterization of Xp21 distal to Duchenne muscular dystrophy (DMD) in the region containing the genes for adrenal hypoplasia congenita (AHC) and glycerol kinase deficiency (GKD) has been limited due to a paucity of probes. Two probes were localized between DXS28 (C7) and AHC, the yeast artificial chromosome insert YHX39 (DXS727) and the polymorphic phage clone QST59 (DXS319). A genomic clone, FT1 (DXS726), $3^{\prime}$ to DMD, was also characterized. Portions of the three probes were sequenced and primer pairs were generated to amplify a sequence-tagged site within each probe. Amplification of DNA from patients confirmed the deletion results obtained by Southern blot analysis, and these three sequence-tagged sites were successfully combined for triplex PCR. In addition to facilitating molecular genetic diagnosis in Xp21, these probes can be used to identify additional YACs and other probes to further increase the genomic information and diagnostic capabilities in this region. (c) 1992 Academic Press, Inc.

\section{INTRODUCTION}

Analyses of DNA from patients with Xp21 contiguous gene syndromes have allowed us to order (Xpter-cen) the following loci: adrenal hypoplasia congenita (AHC), glycerol kinase (GK), and Duchenne muscular dystrophy (DMD) (reviewed in McCabe, 1989). Characterization of Xp21 distal to DMD has been limited due to a paucity of probes.

This project was initiated to improve the mapping information and to develop "sequence-tagged sites" (STSs) (Olson el al., 1989) for the Xp21 region distal to DMD. Experience with DMD has established the diagnostic value of this information, particularly through multiplexing of individual polymerase chain reaction
(PCR) amplifications (Chamberlain et al., 1988). Extending these molecular genetic diagnostic capabilities has considerable clinical importance because of the risk of death due to Addisonian crisis (Kohlschütter et al., 1987) among patients with untreated $A H C$. We report the localization of three probes to this region and the acquisition of sequence information from each. Generation of individual PCR amplifications and a triplex reaction has improved mapping and diagnosis in Xp21 distal to DMD.

\section{MATERIALS AND METHODS}

Patients. A chart indicating the phenotypes of the patients and a summary of the extent of their deletions is shown in Fig. 1. The phenotype designations adapted for microdeletions in this region will be used (McCabe, 1989). These designations describe the known extent of the deletion using the initial onc or two letters of the deleted loci, arranged Xpter to centromere: AGD refers to patients with AHC, GKD, and DMD, and AGK refers to AHC and GKD.

Patients MMC, ESP, GS, JS, JR, MC, MK, CM, and YB have been described previously. MMC, ESP, GS, JS, and MC were described with AGD (Towbin et al., 1989). JR was diagnosed with AGD and also has ophthalmologic features of Oregon eye disease (formerly Åland Island eye disease, Pillers et al., 1990; renamed at HGM 11, Davies et al., 1991). MK was diagnosed with AGD (Francke et al., 1987; Kohlschütter, 1987). CM was reported with AGD (Francke et al., 1987; Towbin et al., 1989; McCabe et al., 1989). YB was diagnosed with AGK (Francke et al., 1987; Marlhens et al., 1987).

TSV has the phenotype of AGD (J. E. Fox). He is developmentally delayed and microcephalic with cryptorchidism, hypotonia, failure to thrive, and cortical atrophy by CT scan. Laboratory studies revealed cortisol levels unresponsive to $\Lambda \mathrm{C} T H$ stimulation, elevated creatine phosphokinase (CPK), and glyceroluria. A lymphocyte karyotype was reported to show an interstitial deletion of Xp21: 46, Y,del(X)(p11.4) (p22.1). This deletion was also detected in the patient's mother, but not in his maternal grandmother. This patient is being maintained on prednisone, Florinef, and a $3-\mathrm{g}$ sodium diet.

CC has AGD, with adrenal insufficiency, elevated CPK, and glyceroluria (W. K. Seltzer and K. P. Corley). He is treated with cortisol and F'lorinef. To our knowledge CC is unrelated to MC.

$T J$ has the AGD phenotype (N. Sakuragawa). At 17 days of age he 
was diagnosed with congenital adrenal hyperplasia and had a high circulating CPK. At 11 months, TJ was diagnosed with complex GKD when he presented with acute encephalopathy after a high fever and a generalized tonic/clonic seizure. He had DMD by muscle biopsy and glyceroluria. CT showed ventricular dilatation and cortical atrophy.

IY is a male with AGD (S. Yamaguchi). Generalized pigmentation was noted shortly after birth. At 22 days of age he was hospitalized for failure to thrive, with hypotonia, vomiting, hyponatremia, hyperkale mia, elevated CPK, and glyceroluria (Yasuda et al., 1990).

LB108 is a 16-year-old male with the AGD phenotype and a large deletion of band Xp21 (G. Romeo). Hypoglycemic crises started at 1 year of age, and he has prominent glyceroluria. He has been wheelchair-bound from the age of 10 , is mentally retarded, and remains prepubertal.

TM has AGD, developmental delay, progressive motor weakness, and adrenal insufficiency (W. B. Rizzo). As an infant he had failure to thrive, poor feeding, emesis, and diarrhea, and he was "floppy." At $6 \frac{7}{12}$ years of age he presented in adrenal insufficiency crisis, with Addisonian pigmentation and markedly elevated $\mathrm{ACTH}$. He was unresponsive to ACTH stimulation and had elevated circulating CPK and glyceroluria. Muscle biopsy was consistent with DMD. A lymphocyte karyotype showed no detectable deletion.

RR has AGK and was referred with the diagnosis of adrenal insufficiency and glyceroluria (P. Brosnan).

DW has isolated AHC (E. R. B. McCabe and G. E. Herman). At 11 months of age he was developmentally delayed, with increased deep tendon reflexes and normal chromosome analysis, serum triglycerides, CPK, and urinary glycerol. An esotropia was noted at 15 months. Previous diagnoses had included primary aldosterone deficiency with cortisone deficiency and congenital adrenal hyperplasia.

TP is a ' $2 \frac{1}{2}$-year-old with isolated AHC (L. G. Biesecker). Chromosome studies, CPK, and urine glycerol were normal. He has been treated with $\mathrm{NaCl}$, glucocorticoids, and mineralocorticoids for $\mathrm{AHC}$ since approximately 3 weeks of age. At $2 \frac{1}{2}$ years of age he was normal developmentally but below the fifth percentile for weight.

$\mathrm{SF}$ was diagnosed with isolated AHC (S. L. Blethen). He has adrenal insufficiency, initially reported to be salt losing only, and is hypertonic. Since 9 months of age he has been treated with both glucocorticoids and mineralocorticoids. SF has normal CPK, normal serum triglycerides, normal development, and no glyceroluria. SF has a maternal male first cousin who presented in the immediate neonatal period with complete adrenal insufficiency.

Markers. Of the previously described probes (Fig. 1), C7 is the most centromeric among L1-4 (DXS68), B24 (DXS67), and C7 (DXS28) (Towbin et al., 1990). The order of L1-4 and B24 has been established using fluorescence in situ hybridization (Trask et al., 1992) and through yeast artificial chromosome (YAC) contigs (Walker et al., 1991)

YHX39 was isolated from a YAC library made from a human-hamster somatic cell hybrid in which the only human DNA was the $X$ chromosome and regionally mapped to Xpter $\rightarrow$ Xp21.2 (Nelson et al., 1991). Alu-vector PCR products were sequenced (Nelson et al., 1991), and primers were generated. The YHX39R primers are 91-92 ATGGT'TCAAGCAT'TTTAGAATACTGC and 91-93 GGTCGTTAAAGGTAAGATCCATGTATGGTTC. This YHX39R primer pair amplifies a 98-bp fragment, DXS727.

The plasmid pQST59M4(b) was derived from the Ch35 clone QST59, which was isolated from the DOE phage library (LAOXNL01) as described (Dietz-Band et al., 1990). Restriction fragments from pQST59 were subcloned and portions of these clones were sequenced. A unique sequence was found in a Tth $111 \mathrm{I}-\mathrm{XbaI}$ fragment, and two primers (3568 TTTTCAATGCAGGAGATGTC and 3569 TGGTTTAGATGGAAGGATAG) that amplify a 229 -bp STS were designed.

FT1 was isolated from a 49 , XXXXY human genomic library and extends $8.8 \mathrm{~kb} 3^{\prime}$ of the human dystrophin gene polyadenylation addition site (Chamberlain, unpublished). This portion of FT1 was subcloned, and a pair of 20 -bp primers were designed for use in PCR (primers 3328 CAATCTAACTGCAAGGAAGG and 3329 TATGGAGTGAAGAACCTCTG). These primers amplify a 283-bp STS, DXS726.
PCR. PCR was carried out in $50 \mu \mathrm{l}$ containing $10 \mathrm{mM}$ Tris $-\mathrm{HCl}$, pH $8.3,50 \mathrm{mM} \mathrm{KCl}, 1.5 \mathrm{mM} \mathrm{MgCl}_{2}, 0.001 \%$ gelatin, $2 \mu M$ of each primer, $200 \mu M$ each dNTP, 1.4 units of Taq polymerase, and $200 \mathrm{ng}$ of genomic DNA. Reactions were covered with a $50-\mu \mathrm{l}$ mineral oil overlay. Samples were denatured at $95^{\circ} \mathrm{C}$, and the enzyme was added at $94^{\circ} \mathrm{C}$ and subjected to $25-35$ cycles: $94^{\circ} \mathrm{C} 1 \mathrm{~min}, 50-55^{\circ} \mathrm{C} 1 \mathrm{~min}$, and $72^{\circ} \mathrm{C} 1 \mathrm{~min}$, with a final $7 \mathrm{~min}$ at $72^{\circ} \mathrm{C}$. The optimum annealing temperature is $50^{\circ} \mathrm{C}$ for primers 91.92 and $91-93$ (YHX39) and primers 3568 and 3569 (QST59) and $55^{\circ} \mathrm{C}$ for primers 3328 and 3329 (FT1). Multiplex reactions with all three sets of primers used $50^{\circ} \mathrm{C}$. Blank (no DNA), positive control (normal human DNA), and negative control (patient with a large Xp chromosome deletion) were included with each set of reactions.

\section{RESULTS}

Eighteen patients were characterized, and the results are shown in Fig. 1. The YHX39R primer pair amplified a 98-bp fragment, DXS727, which mapped the same as YHX39L by Southern blot in the patients (Fig. 1). The PCR results are shown in Fig. 2. The interval between C7 (DXS28) and YHX39R (DXS727) contained the telomeric deletion breakpoints of several patients, including MC, CC, TJ, MK1753, CM, IY, and YB.

The presence of the DXS319 locus in the patients was determined by Southern hybridization with the probe pQST59M4(b) (Fig. 1). The QST59 (DXS319) primers amplified a 229-bp STS, and the PCR results are shown in Fig. 2. The QST59 STS defined a difference in the telomeric deletion breakpoints between patients TM and LB108: the telomeric deletion breakpoint for both patients was centromeric of YHX39 (DXS727), but only the telomeric deletion breakpoint for TM was centromeric of QST59, presumably between DXS319 and the centromeric extent of $\mathrm{AHC}$.

FT1 was sequenced, and primers that amplified a 283 bp STS (DXS726) were designed. The PCR results are shown in Fig. 2.

To improve the diagnostic efficiency for diagnosis of patients with deletions involving this region, the three sets of PCR primers were combined into one multiplex reaction (Fig. 2). The results were identical to those seen in the uniplex reactions (data not shown).

\section{DISCUSSION}

Patients with deletions who have AHC, but not GKD or DMD, have been described (Yates et al., 1987; and DW this paper). These patients have also had developmental delay, in contrast to those with isolated $\mathrm{AHC}$ and no detectable deletions (TP,SF) who are developmentally normal and presumably have point or other small mutations. Patients with GKD and DMD who do not have AHC (Davies et al., 1988) and patients who have AHC and GKD but not DMD (Marlhens et al., 1987, Franke et al., 1987; and RR this paper) have been described. Analyses of DNA from these patients with Xp21 contiguous gene syndromes have allowed the loci to be ordered (Xpter-cen): AHC, GK, and DMD (reviewed in McCabe, 1989). 


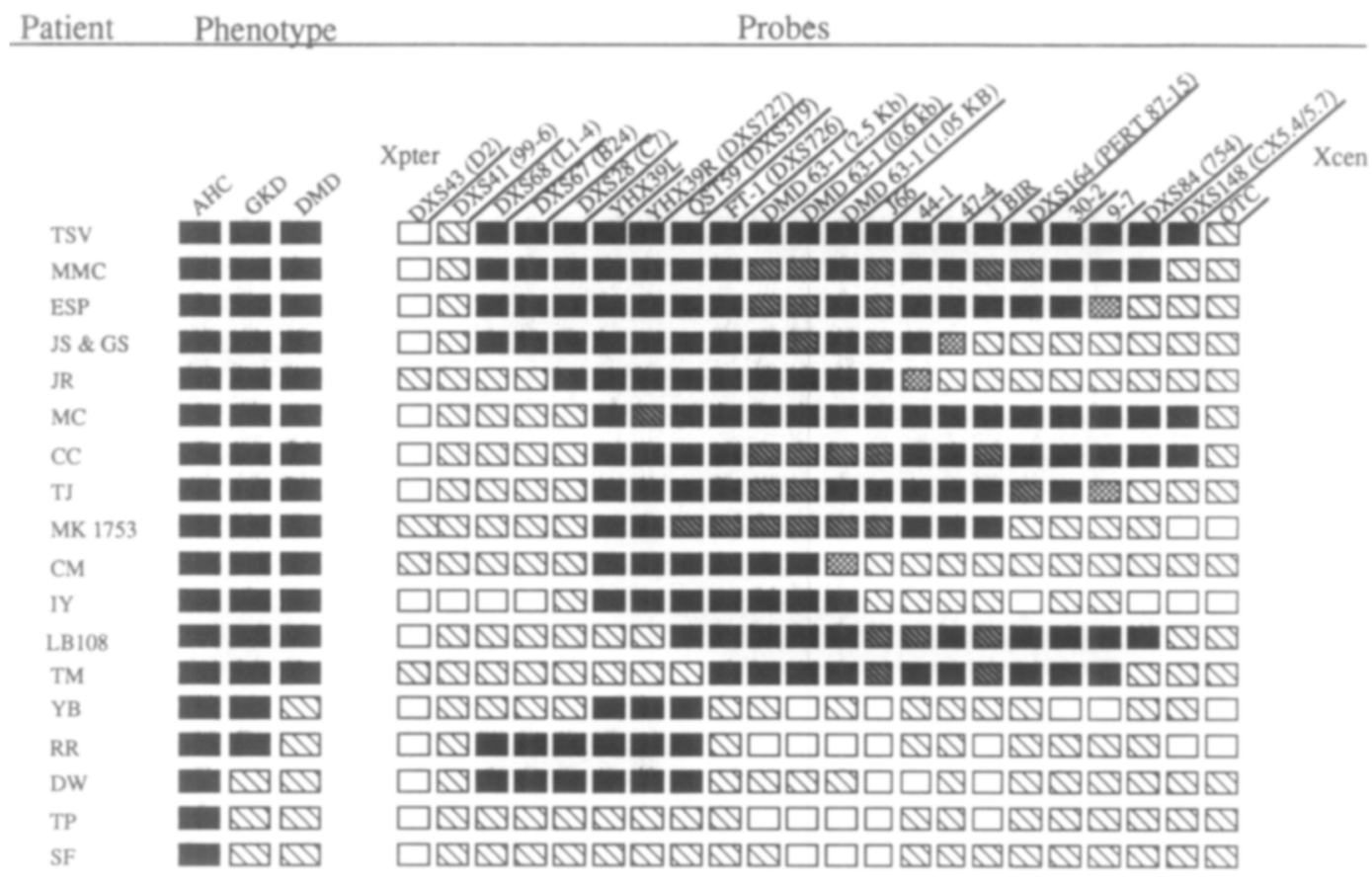

FIG. 1. Summary of patient phenotypes and molecular genetic results. $\mathbf{n}$, Presence of phenotypic features and absence of hybridization or amplification of probe (whichever applies). . . Absence of phenotypic features and hybridization or amplification of probe (whichever applies). 诸, A partial or a junction fragment. and $\square$, Probes not tested and presumed deleted or present, respectively. The genomic probes (Towbin et al., 1989; McCabc et al., 1989; Francke et al., 1987) and the DMD cDNA probes (McCabe ct al., 1989) have been described previously.

Three markers were localized in Xp21 distal to DMD, between DXS28 (C7) and DMD. The PCR results for these markers, YHX39, QST59, and FT1, confirm the mapping results obtained by Southern blotting. Analysis of DNA from patients mapped these probes (Fig. 3) with deletion breakpoints in each of these intervals: DXS28 (C7) to YHX39, YHX39 to QST59, QST59 to AHC, and GK to FT1. Therefore, the genes for AHC and GK are flanked by the markers QST59 and FT1. YHX39 and QST59 are located between DXS28 (C7) and AHC, with YHX39 telomeric to QST59. FT1 is between GK and DMD.

One other probe has been mapped to the region between DXS28 (C7) and DMD. This probe, JC-1, was cloned from the junction fragment of a patient with

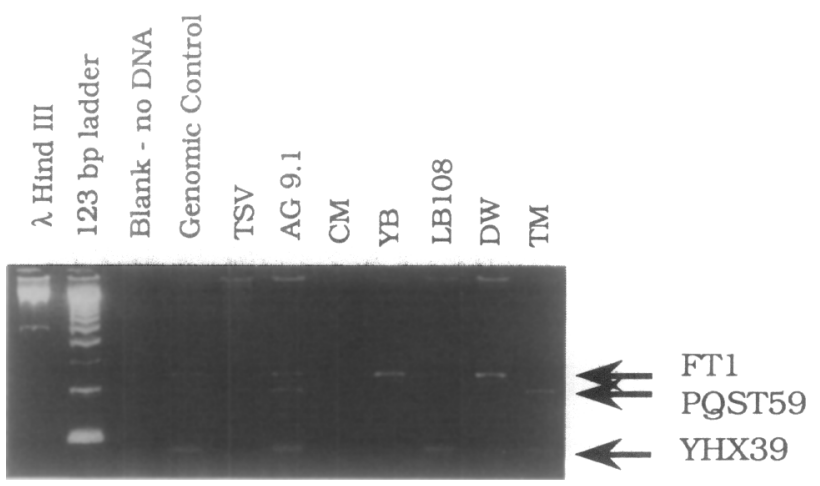

FIG. 2. Results of multiplex PCR amplification of patient DNAs using primers $91-92,91-93,3568,3569,3328$, and 3329 . The top band is FT1 (283 bp), the middle band is QST59 (229 bp), and the bottom band is YHX39R (98 bp).
DMD and GK, but not AHC (Love et al., 1990). JC-1 maps to the region between the centromeric extent of the AHC and the centromeric extent of the GK genes (Love et al., 1990). The patient deletion breakpoints and the new probes (YHX39, QST59, and FT1) can be used as additional landmarks to expand the map in the region (Fig. 3). The orientation of YHX39R and YHX39L is determined by YAC mapping data (Worley et al., in preparation). When a complete YAC contig is developed for this region, all of the patient deletion breakpoints will be separated unless there is some feature of the DNA that encourages chromosome rearrangement.

The patient deletion breakpoints define intervals that indicate more precisely the location of the genes in the region (Fig. 3). For instance, patient TM has a deletion that includes or interrupts the $\mathrm{AHC}$ gene and extends toward the centromere. DW has a deletion that includes or interrupts the AHC gene and extends toward the telomere, but does not interrupt the GK gene. Therefore, some or all of the interval between these two breakpoints must be within the AHC gene. Likewise, the interval between the $\mathrm{DW}$ centromeric deletion breakpoint (or the probe $\mathrm{JC}-1$ ) and the $\mathrm{YB}$ and $\mathrm{RR}$ centromeric deletion breakpoints contains all or part of the GK gene.

The patient information and the new markers are important in terms of the diagnostic abilities in the region. The three new STSs have been combined into a multiplex PCR reaction for DNA diagnosis. Several patients have had phenotypes suggesting the extension of deletions in a telomeric direction beyond $\mathrm{DMD}$, but previously available markers were unable to provide molecular genetic evidence to support this. The markers re- 

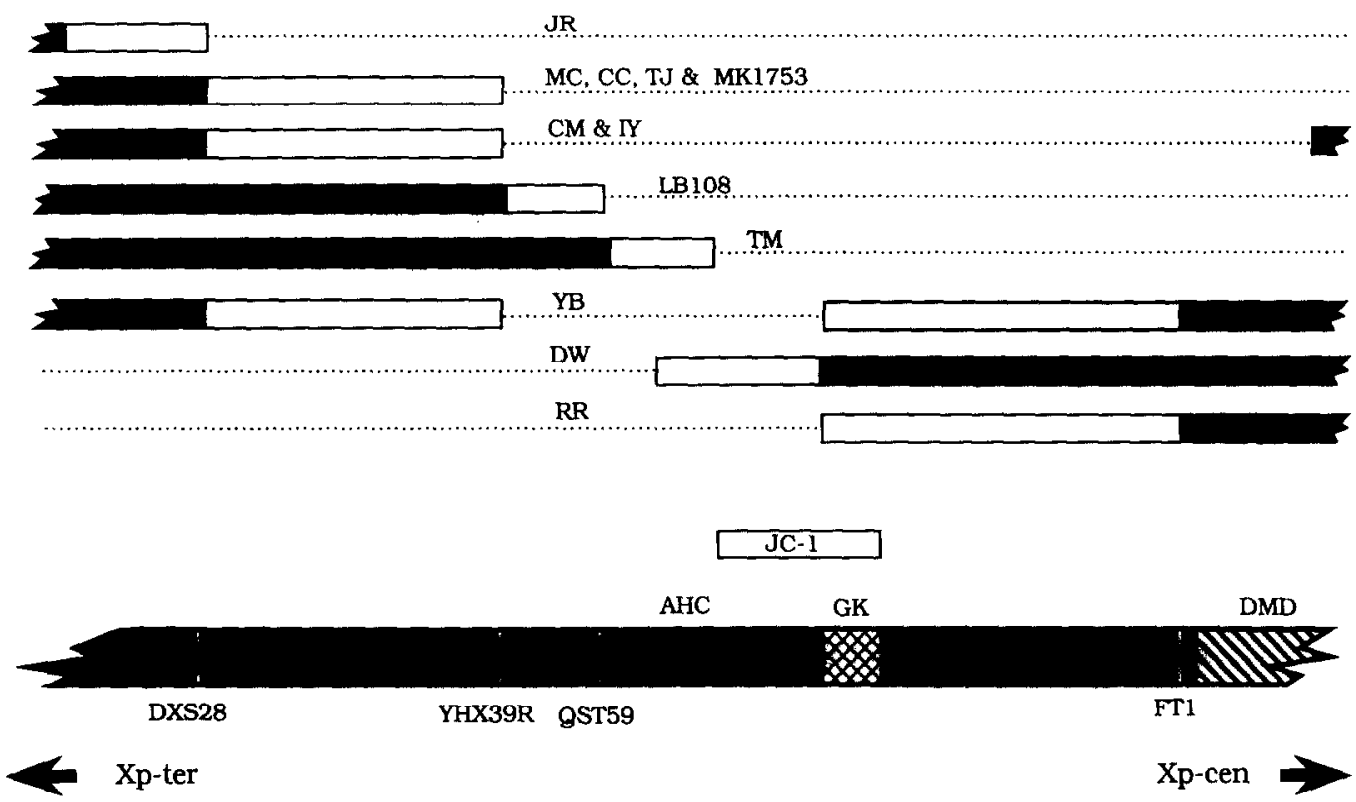

FIG. 3. Map of the region in Xp21 between C7 (DXS28) and FT1 (no scale). The upper figures are maps of the patients' deletions, $\mathbf{n}$, Parts of the chromosome that are present; . . ., deleted regions; and $\square$, uncertainty in the location of the deletion breakpoints. (Bottom) Filled bars

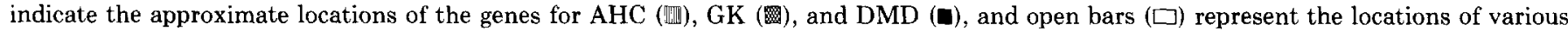
probes. The probe JC-1 (McCabe, 1989) has not been mapped in these patients. JC-1 may be located anywhere between the centromeric extent of the $\mathrm{AHC}$ gene and the centromeric extent of the GK gene as shown.

ported here provide that evidence for patients $\mathrm{MC}, \mathrm{CC}$, TJ, MK1753, CM, IY, LB108, and TM. In addition, one patient, YB, with AGK (AHC, GKD) whose DNA had not shown a deletion with any previous probes, was shown to have a deletion by these markers.

In conclusion, we have characterized three probes (YHX39, QST59, and FT1) and their corresponding STSs that map between DXS28 (C7) and DMD as shown. This study identified a new marker (QST59), which is the closest flanking marker to AHC. This has enabled better definition of the breakpoint for patient LB108, which flanks and significantly narrows the interval in which the AHC gene is located. The fact that both QST59 and YHX39 identify a previously undetectable deletion in the patient YB confirms that QST59 is significantly closer to AHC than previously available probes. This indicates the value of these new markers, both for the diagnosis of AGD and for the cloning of the $\mathrm{AHC}$ and GK genes.

\section{ACKNOWLEDGMENTS}

The authors acknowledge the contribution of G. E. Herman, M.D., Ph.D. (Baylor College of Medicine), and K. P. Corley, M.D. (Omaha Children's Hospital), for contributing information and blood from DW and CC, respectively. This work was supported by grants from the National Institute of Child Health and Human Development, National Institutes of Health (5 R01 HD22563; 5 P30 HD24064, the Baylor Mental Retardation Research Center; and 5 P30 HD27823, the Baylor Child Health Research Center), and a grant (1 P50 HG00210) for the Baylor College of Medicine Human Genome Program Center from the National Center for Human Genome Research.

\section{REFERENCES}

Chamberlain, J. S., Gibbs, R. A., Ranier, J. E., Nguyen, P. N., and Caskey, C. T. (1988). Deletion screening of the Duchenne muscular dystrophy locus via multiplex DNA amplification. Nucleic Acids Res. 16: 141-155.

Davies, K. E., Mandel, J.-L., Monaco, A. P., Nussbaum, R. L., and Willard, H. F. (1991). Report of the committee on the genetic constitution of the X chromosome. Cytogenet. Cell Genet. 58: 853-966.

Davies, K. E., Patterson, M. N., Kenwrick, S. J., Bell, M. V., Sloan, H. R., Westman, J. A., Elsas II, L. J., and Mahan, J. (1988). Fine mapping of glycerol kinase deficiency and congenital adrenal hypoplasia within Xp21 on the short arm of the human X chromosome. Am. I. Med. Genet. 29: 557-564.

Dietz-Band, J. N., Turico, A. E., Willard, H. F., Vincent, A., Skolnick, M. H., and Barker, D. F. (1990). Isolation, characterization, and physical localization of 33 human X chromosome RFLP markers. Cytogenet. Cell. Genet. 54: 137-141.

Francke, U., Harper, J. F., Darras, B. T., Cowan, J. M., McCabe, E. R. B., Kohlschütter, A., Seltzer, W. K., Saito, F., Goto, J., Harpey, J. P., and Wise, J. E. (1987). Congenital adrenal hypoplasia, myopathy, and glycerol kinase deficiency: Molecular genetic evidence for deletions. Am. J. Hum. Genet. 40: 212-227.

Kohlschütter, A., Willig, H. P., Schlamp, D., Kruse, K., McCabe, E. R. B., Schafer, H. J., Bechenkamp, G., and Rohkamm, R. (1987). Infantile glycerol kinase deficiency-a condition requiring prompt identification. Eur. J. Pediatr. 146: 575-581.

Love, D. R., Bloomfield, J. F., Kenwrick, S. J., Yates, J. R. W., and Davies, K. E. (1990). Physical mapping distal to the DMD locus. Genomics 8: 106-112.

Marlhens, F., Chelly, J., Kaplan, J. C., Lefrancois, D., Harpey, J. P., and Dutrillaux, B. (1987). Familial deletion of Xp21.2 with glycerol kinase deficiency and congenital adrenal hypoplasia. Hum. Genet. 77: 379-383.

McCabe, E. R. B. (1989). Disorders of glycerol metabolism. In "The Metabolic Basis of Inherited Disease" (C. R. Scriver, A. L. Beaudet, W. S. Sly, and D. Valle, Eds.), 6th ed., pp. 945-961, McGraw-Hill, New York.

McCabe, E. R. B., Towbin, J., Chamberlain, J. C., Baumbach, L., Wit kowski, J., van Ommen, G. J. B., Koenig, M., Kunkel, L. M., and Seltzer, W. K. (1989). Complementary DNA probes for the Duchenne muscular dystrophy locus demonstrate a previously undetectable deletion in a patient with dystrophic myopathy, glycerol 
kinase deficiency, and congenital adrenal hypoplasia. J. Clin. Invest. 83: 95-99.

Nelson, D. L., Ballabio, A., Victoria, M. F., Pieretti, M., Bies, R. D., Gibbs, R. A., Maley, J. A., Chinault, A. C., Webster, T. D., and Caskey, C. T. (1991). Alu PCR for regional assignment of 110 yeast artificial chromosome clones from the human X chromosome: Identification of clones associated with a disease locus. Proc. Natl. Acad. Sci. USA 88: 6157-6161.

Olson, M., Hood, L., Cantor, C., and Botstein, D. (1989). A common language for physical mapping of the human genome. Science $\mathbf{2 4 5}$ : $1434-1435$.

Pillers, D. M., Weleber, R. G., Powell, B. R., Hanna, C. F., Magenis, R. E., and Buist, N. R. M. (1990). Åland Island eye disease (ForsiusEriksson ocular albinism) and an Xp21 deletion in a patient with Duchenne muscular dystrophy, glycerol kinase deficiency, and congenital adrenal hypoplasia. Am. J. Med. Genet. 36: 23-28.

Towbin, J. A., Wu, D., Chamberlain, J. S., Larsen, P. D., Seltzer, W. K., and McCabe, E. R. B. (1989). Characterization of patients with glycerol kinase deficiency utilizing cDNA probes for the Duchenne muscular dystrophy locus. Hum. Genet. 83: 122-126.

Towbin, J. A., Chamberlain, J. S., Wu, D., Pillers, D. M., Seltzer, W. K., and McCabe, E. R. B. (1990). DXS28 (C7) maps centromeric to DXS68 (L1-4) and DXS67 (B24) by deletion analysis. Genomics 7: 442-444.

Trask, B. J., Massa, H. F., and Burmeister, M. (1992). Fluorescence in situ hybridization establishes the order cen-DXS28(C7)DXS67(B24)-DXS68(L1)-tel in human chromosome Xp21.3.Genomics 13: 455-457.

Walker, A. P., Larin, Z., Lehrach, H., and Monaco, A. P. (1991). Human Xp21 YAC contig maps. Cytogenet. Cell Genet. 58: A27093.

Yasuda, K., Kondo, T., Hiraizumi, Y., Fujii, H., and Yamazaki, M. (1990). A case with complex glycerol kinase deficiency diagnosed during the newborn period. Med. Genet. Res. 12: 11-19.

Yates, J. R. W., Gillard, G. F., Cooke, A., Evans, T. J., and FergusonSmith, M. A. (1987). Localisation of the gene for congenital adrenal hypoplasia. J. Med. Genet. 24: 241. 